\title{
Heterogeneity of Polyneuropathy Associated with Anti-MAG Antibodies
}

\author{
Laurent Magy, ${ }^{1}$ Raphaël Kaboré, ${ }^{1}$ Stéphane Mathis, ${ }^{2}$ Prisca Lebeau, ${ }^{1}$ Karima Ghorab, ${ }^{1}$ \\ Christiane Caudie, ${ }^{3}$ and Jean-Michel Vallat ${ }^{1}$ \\ ${ }^{1}$ Department of Neurology, Centre de Référence "Neuropathies Périphériques Rares", CHU Limoges, 2 Avenue Martin Luther-King, \\ 87042 Limoges, France \\ ${ }^{2}$ Department of Neurology, CHU Poitiers, University of Poitiers, 2 Rue de la Milétrie, 86021 Poitiers, France \\ ${ }^{3}$ Centre de Biologie et de Pathologie Est, Hospices Civils de Lyon, 69000 Lyon, France
}

Correspondence should be addressed to Jean-Michel Vallat; jean-michel.vallat@unilim.fr

Received 4 November 2014; Accepted 7 April 2015

Academic Editor: Douglas C. Hooper

Copyright (C) 2015 Laurent Magy et al. This is an open access article distributed under the Creative Commons Attribution License, which permits unrestricted use, distribution, and reproduction in any medium, provided the original work is properly cited.

\begin{abstract}
Polyneuropathy associated with IgM monoclonal gammopathy and anti-myelin associated glycoprotein (MAG) antibodies is an immune-mediated demyelinating neuropathy. The pathophysiology of this condition is likely to involve anti-MAG antibody deposition on myelin sheaths of the peripheral nerves and it is supposed to be distinct from chronic inflammatory demyelinating neuropathy (CIDP), another immune-mediated demyelinating peripheral neuropathy. In this series, we have retrospectively reviewed clinical and laboratory findings from 60 patients with polyneuropathy, IgM gammopathy, and anti-MAG antibodies. We found that the clinical picture in these patients is highly variable suggesting a direct link between the monoclonal gammopathy and the neuropathy. Conversely, one-third of patients had a CIDP-like phenotype on electrodiagnostic testing and this was correlated with a low titer of anti-MAG antibodies and the absence of widening of myelin lamellae. Our data suggest that polyneuropathy associated with anti-MAG antibodies is less homogeneous than previously said and that the pathophysiology of the condition is likely to be heterogeneous as well with the self-antigen being MAG in most of the patients but possibly being another component of myelin in the others.
\end{abstract}

\section{Introduction}

Ten percent of patients with a polyneuropathy of unknown cause have a monoclonal gammopathy [1]. Most of these patients have an IgM dysglobulinemia and around 70\% of those have anti-myelin associated glycoprotein (MAG) antibody detected by enzyme-linked immunosorbent assay (ELISA). Indeed, it has long been demonstrated that MAG behaves as a self-antigen in patients with polyneuropathy and IgM monoclonal gammopathy [2]. In the past 25 years, numerous series have described anti-MAG neuropathy as a homogeneous entity $[3,4]$. The clinical picture of the disorder usually consists of a chronic sensory polyneuropathy with ataxia and tremor of progressive worsening. Motor involvement, if present, usually occurs lately in the course of the disorder [5]. Nerve conduction studies display a demyelinating pattern with distally accentuated slowing of motor conduction, no conduction block, and a severe reduction of sensory nerve action potentials (SNAPs) [6]. When nerve biopsy is performed, it shows signs of demyelination on semithin sections and teased fiber studies, and electron microscopic examination usually displays the classic pattern of widening of myelin lamellae (WML), which is considered the pathological hallmark of the disease [7]. This latter feature corresponding to deposits of the monoclonal IgM on myelin sheath distinguishes pathologically anti-MAG neuropathy from chronic inflammatory demyelinating polyradiculoneuropathy (CIDP) [8].

In the present study, we show that anti-MAG neuropathy is indeed a heterogeneous disorder as demonstrated by 
careful clinical, electrophysiological, and neuropathological analysis. We discuss the potential reasons for this heterogeneity and its therapeutic implications.

\section{Patients and Methods}

The data from all patients with a polyneuropathy associated with an IgM monoclonal gammopathy and anti-MAG antibodies seen in our neurology department over the previous 25 years were retrospectively reviewed.

2.1. Clinical Findings. Age, gender, and duration of symptoms at the time of diagnosis were extracted from the medical charts. Based on clinical evaluation, patients were classified as having pure sensory neuropathy, ataxia with sensory neuropathy, and sensorimotor neuropathy. Ataxia was considered if patients had a positive Romberg sign, subjective impression of balance loss, and visible balance disturbance when walking. A sensorimotor neuropathy was defined by the presence of sensory loss on clinical examination and motor weakness at 4 or less on the Medical Research Council (MRC) scale in any limb segment (except if patients had weakness only in toe extensors).

2.2. Electrodiagnostic Studies. At the time of referral, 56 (93\%) patients had nerve conduction studies performed in our neurophysiology department as described [9]. Bilateral motor conduction studies of median, ulnar, peroneal, and tibial nerves and sensory conduction studies of sural, median, and ulnar nerves were performed. The nerve conduction data were considered sufficient for analysis when at least 2 motor nerves and one sensory nerve were examined in the lower limbs and 2 motor nerves and 2 sensory nerves in the upper limbs. Partial conduction block was defined by a reduction of compound muscle action potential (CMAP) by proximal stimulation of at least $50 \%$ in the lower limb and at least $30 \%$ in the upper limb. Temporal dispersion was defined by a lengthening of CMAP of at least $30 \%$ by proximal stimulation. The terminal latency index (TLI) was calculated for the median and ulnar nerves as described [10]. For the purpose of this study, patients were retrospectively classified as having typical anti-MAG neuropathy: a grossly symmetric demyelinating neuropathy with distally predominant demyelination based on low terminal latency indexes, a severe decrease of sensory nerve action potential (SNAP) amplitudes in the lower limbs, and no conduction block or temporal dispersion [11], or a CIDP-like pattern: fulfillment of the EFNS/PNS criteria for CIDP [12], normal TLI in ulnar and median nerves and/or presence of conduction block and/or temporal dispersion in at least one nerve, and/or normal or near normal SNAPs in the lower limbs.

2.3. Biological Studies. All patients had IgM monoclonal gammopathy determined by serum immunoelectrophoresis or immunofixation. Search for anti-MAG antibodies was done several years ago for 4 patients by immunochromatography. Sera from most of the patients were tested for antiMAG antibodies by ELISA (Bühlmann laboratories AG,
Schonenbuch, Switzerland). The cut-off positive value was considered above 1000 BTU (Bühlmann Titer Unit) according to the manufacturer's instructions [13]. Other sera were tested in the center of biology and pathology (Hospices Civils de Lyon) for anti-MAG antibodies and for antibodies to sulfate-3-glucuronyl paragloboside. To confirm anti-myelin activity, we performed indirect immunofluorescence with the sera from 45 patients on normal human peripheral nerve as described earlier [14]. In 15 patients, we tested for the presence of anti-glycolipid antibodies as described previously [15].

2.4. Nerve Biopsy. Forty-three of the 60 patients underwent nerve biopsy after informed consent. Depending on clinical and/or neurophysiological involvement, the sural, superficial peroneal or sensory branch of a radial nerve was taken. One fragment was fixed in $10 \%$ formaldehyde and embedded in paraffin. Sections were stained using conventional methods. Other fascicles were fixed in buffered glutaraldehyde and then embedded in Epon. Several blocks were used for semithin sections subsequently colored with toluidine blue. Ultrathin sections were prepared as described [14] and viewed in a JEOL electron microscope. A third fragment was frozen for direct immunofluorescence (DIF) using specific antisera for IgG, IgA, IgM, lambda and kappa light chains, and C3d conjugated to fluorescein, to reveal immunoglobulin deposits on the patients' nerves.

2.5. Statistical Analysis. The clinical and laboratory data were collected and analyzed with the SPSS 8.0 software. Qualitative data were expressed as percentages with their 95\% confidence interval and quantitative data were expressed as mean \pm SD. Statistical significance of differences between categorical variables was determined by means of $\chi^{2}$ or Fisher exact test as appropriate and for differences between continuous variables by means of Student's $t$-test. Statistical significance for all analyses was defined as $p<0.05$.

\section{Results}

The main clinical and laboratory data are summarized in Table 1.

3.1. Demographic and Clinical Data. There were 60 patients (43 males: $72 \%$ ). Mean age at study was $67 \pm 10$ years (range 46-87) and mean duration of symptoms at diagnosis was $35 \pm$ 44 months (range 2-204). Nineteen (32\%) patients had the distal acquired demyelinating symmetrical (DADS) phenotype [16]. Symptoms and signs in these patients were mostly sensory and proximal segments of limbs were never involved by sensory and/or motor signs. These patients had usually numbness or tingling limited to the feet and prominent ataxia and 3 of them had additional pain. Tendon reflexes were abolished at least in the lower limbs in these patients. Seventeen $(28 \%)$ patients had isolated distal numbness or pain without ataxia. Pain had the classical characteristics of neuropathic pain [17] in these patients (mainly burning, squeezing, and painful cold) and was a prominent symptom in seven of those who used various analgesics (clonazepam, 


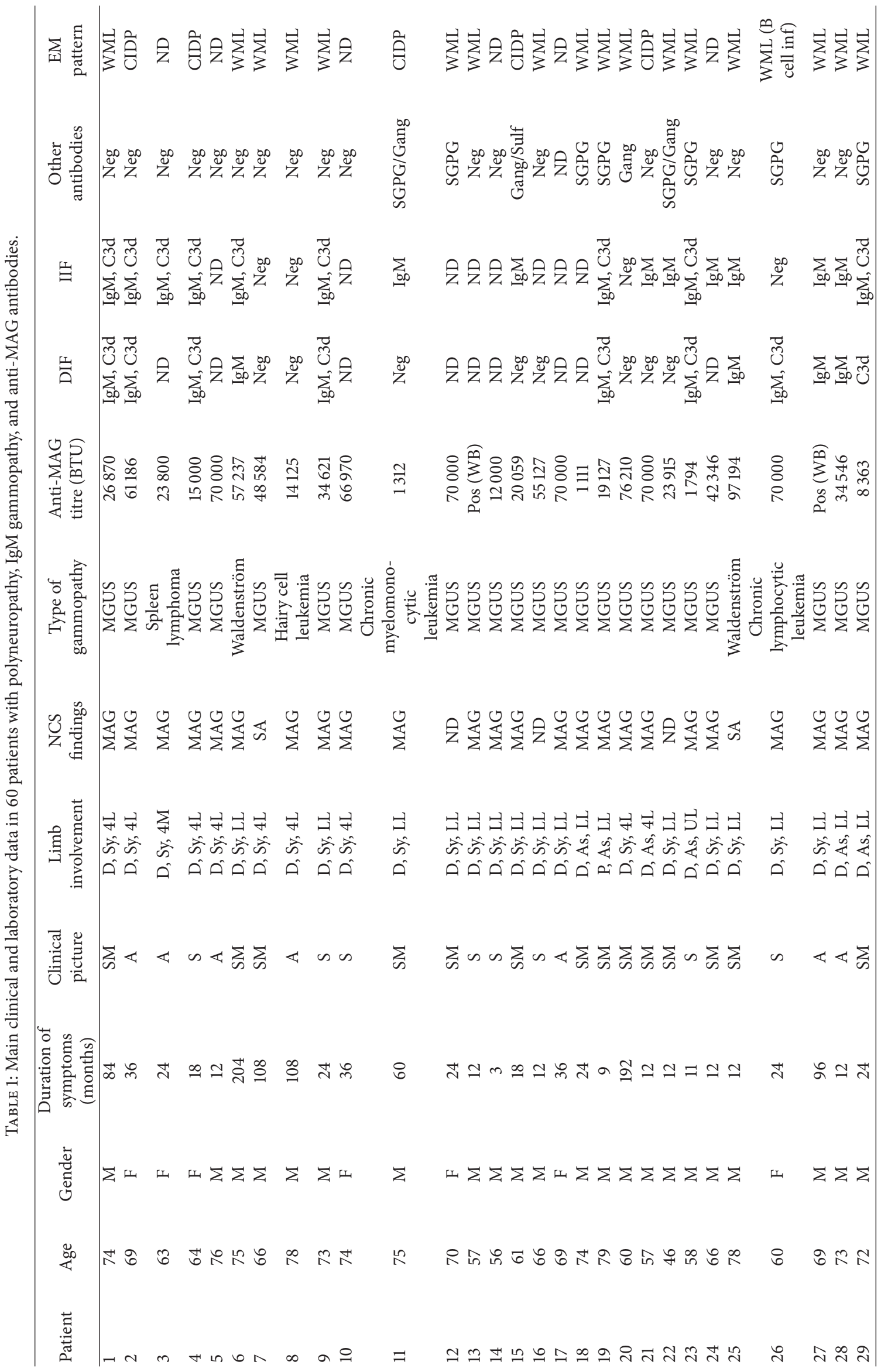




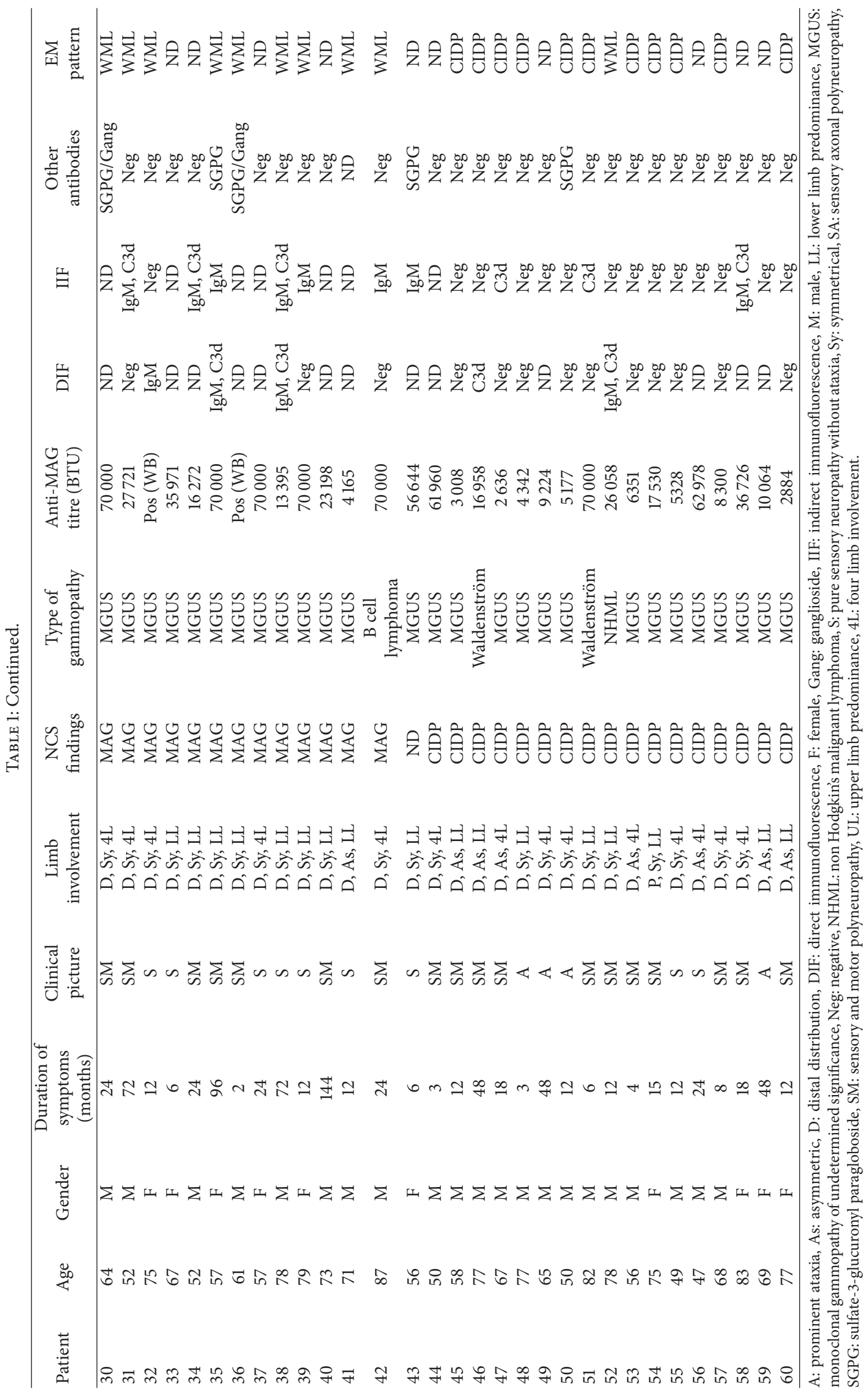


gabapentin, amitriptyline, and pregabalin). Physical examination showed distal hypesthesia and tendon reflexes were variably impaired in this subgroup of patients (ranging from diminished Achilles reflexes to abolition of reflexes in all four limbs). 32 (53\%) patients had variable degrees of motor weakness leading to severe impairment in a minority of them. Weakness was usually distal although 2 patients had prominent proximal weakness. Mean duration of symptoms at diagnosis was 45.6 months in patients with sensorimotor neuropathy and 31.9 months in other patients although this difference did not reach significance. The presence of any weakness was not statistically linked with the duration of symptoms when comparing patients with duration of disease below or above two years.

3.2. Nerve Conduction Studies. Fifty-six patients had exploitable nerve conduction studies. 37 (66\%) patients had the typical anti-MAG pattern as defined above [11]. Most of them had a severe reduction of SNAPs in all four limbs, a distal pattern of demyelination as defined by low TLIs in at least one nerve, and no conduction block. 17 (30\%) patients had nerve conduction studies consistent with CIDP [12]. Most of these patients had conduction block and/or marked temporal dispersion and some had normal or near normal SNAPs in the lower limbs. The two remaining patients had a sensory axonal neuropathy. There was no correlation between the different clinical subtypes and the electrodiagnostic pattern of nerve involvement.

3.3. Biological Data. All patients had IgM monoclonal gammopathy and anti-MAG antibodies. For 51 (85\%) patients, the diagnosis was of a monoclonal gammopathy of undetermined significance (MGUS) although 4 patients had Waldenström's macroglobulinemia, 2 patients had non-Hodgkin's malignant lymphoma, and 3 patients had chronic leukemia. One patient with chronic lymphocytic leukemia had epineurial B cell infiltrates suggestive of lymphomatous transformation (see below). The titer of anti-MAG antibodies was highly variable among patients, ranging from $1111 \mathrm{BTU}$ to > $70000 \mathrm{BTU}$ (Table 1). Although quite strongly correlated with the neuropathological pattern and the electrodiagnostic findings, the biological data were not correlated with the clinical pattern as defined above.

3.4. Neuropathology and Indirect Immunofluorescence Studies. Forty-three patients had undergone nerve biopsy. All nerve samples showed a decrease in myelinated fiber density on semithin sections. This axonal loss was highly variable among patients, ranging from slight to severe. Among patients who underwent nerve biopsy, 26 (60\%) had typical widening of myelin lamellae (WML) on electron microscopic examination (Figure 1). WML usually concerned a minority (5$10 \%)$ of large myelinated axons. Most of these patients had mild cellular infiltrates composed of lymphocytes (antiCD45 staining) and macrophages (anti-CD68 staining): 8 of these had macrophage-associated demyelination, a feature considered typical of CIDP (Figure 2). One of the female patients (26) with MGUS and WML had also prominent B cell

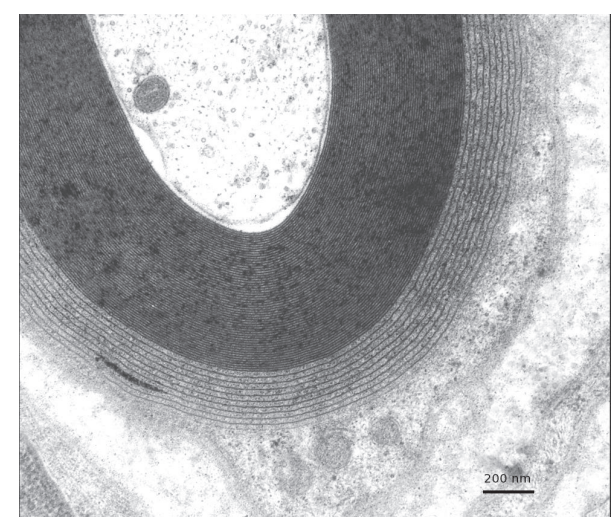

Figure 1: Typical widening of the most external myelin lamellae. Electron micrograph. Transverse section.

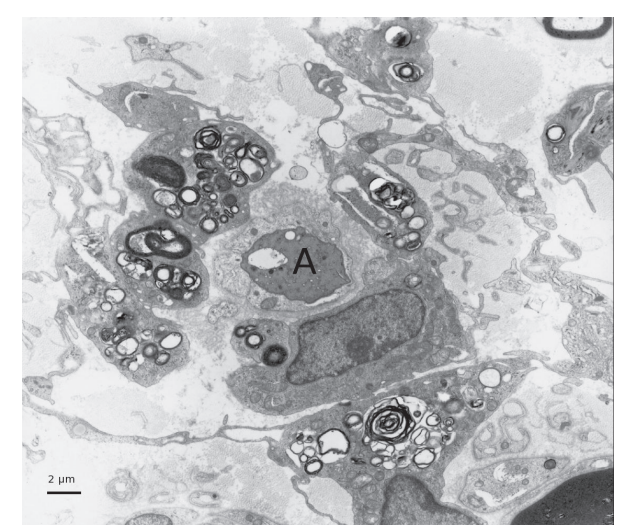

FIGURE 2: A naked axon (A) is surrounded by several macrophages loaded with myelin debris in a patient with the CIDP-like profile. Electron micrograph, transverse section.

infiltrates in the epineurium (anti-CD20 staining) suggesting the conversion of the gammopathy to a B cell lymphoma (Figure 3). Seventeen (40\%) of the patients who underwent nerve biopsy had no WML. All of these patients had features typical of a demyelinating neuropathy with onion bulb formations and/or completely demyelinated axons (Figure 4). Pictures of remyelinating axons with abnormally thin myelin sheaths were frequent. Additionally, macrophage-associated demyelination was encountered in all nerve samples from these patients. Thirty-five patients had indirect immunofluorescence (IIF) to test reactivity of their serum against normal nerve. IIF failed to show any staining with anti-IgM and/or anti-C3D in 9 patients and was mostly negative in patients with the CIDP pattern and a low titer of anti-MAG antibodies. Nerve biopsy findings were not correlated with any clinical subtype as defined above.

3.5. Correlations according to the Electrodiagnostic and Neuropathological Status. The details of the two subpopulations of patients according to the electrodiagnostic pattern are summarized in Table 2. Among the 37 patients who had the typical anti-MAG pattern on nerve conduction studies, 27 had nerve biopsy, of which 21 (78\%) showed typical WML 
TABLE 2: Main clinical and laboratory data from 2 subpopulations of patients with polyneuropathy and anti-MAG antibodies according to nerve conduction findings.

\begin{tabular}{lccc}
\hline & $\begin{array}{c}\text { Patients with the anti-MAG } \\
\text { pattern } \\
n=37\end{array}$ & $\begin{array}{c}\text { Patients with the CIDP-like } \\
\text { pattern } \\
n=17\end{array}$ & Statistical significance \\
\hline Typical widening of myelin lamellae & $21 / 27(78 \%)$ & $2 / 12(17 \%)$ & $p<0.05$ \\
Positive indirect immunofluorescence & $20 / 22(91 \%)$ & $3 / 10(30 \%)$ & $p<0.05$ \\
Anti-MAG antibodies $>10$ 000 BTU & $32 / 37(86 \%)$ & $7 / 17(41 \%)$ & $p<0.05$ \\
Mean titer of anti-MAG antibodies (SD) & $38410(26626)$ & $20560(23097)$ & $n<0.05$ \\
\hline
\end{tabular}

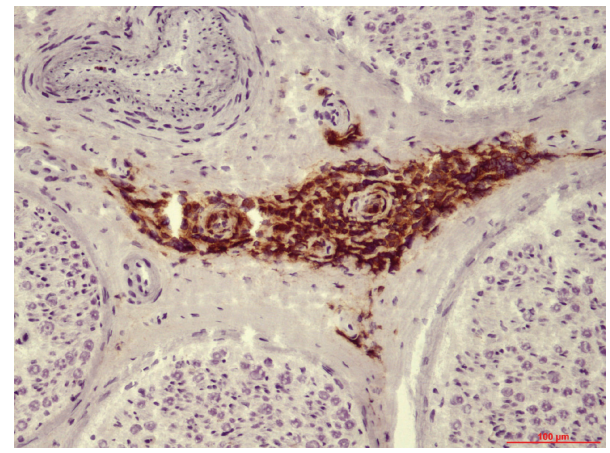

Figure 3: Several epineurial vessels are surrounded by prominent B cell infiltrates (patient 26). Paraffin transverse section labelled with anti-CD20 antibody.

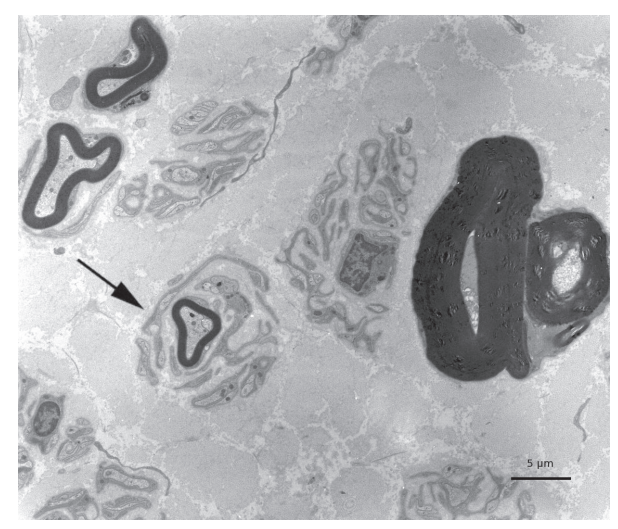

Figure 4: A myelinated axon is surrounded by onion bulb formations in a patient with the CIDP-like profile. Electron micrograph, transverse section.

$(p<0.05)$. Twenty-two of these 37 patients had IIF, which was positive in 20 (91\%) cases. Of note, anti-MAG antibodies were above $10000 \mathrm{BTU}$ in $32(86 \%)$ of the 37 patients with NCS suggestive of anti-MAG neuropathy $(p<0.05)$.

Among the 17 patients who had nerve conduction studies consistent with CIDP, 12 underwent nerve biopsy, which showed histological abnormalities suggestive of CIDP, without WML on electron microscopic examination in 10 (83\%) cases $(p<0.05)$. Ten of these 17 patients had IIF, which was negative in 7 cases. Moreover, 10 of these 17 patients had antiMAG antibody titer below 10000 BTU $(p<0.05)$.
Four patients who had concordant nerve conduction and neuropathological findings suggestive of anti-MAG neuropathy had low titers of anti-MAG antibodies (1111-8663 BTU) but positive SGPG antibodies. IIF was carried out in 3 of these 4 patients and was positive in all.

\section{Discussion}

The objective of this retrospective study was to reexamine the concept that polyneuropathy associated with antiMAG antibodies is a homogeneous entity. This syndrome is usually characterized by a slowly progressing sensory ataxic neuropathy. Nerve conduction studies usually show a symmetrical demyelinating neuropathy with distal accentuation and the phenotype is sometimes referred to as distal acquired demyelinating symmetric neuropathy (DADS) [16]. The demographic features of our patients (mean age at diagnosis of 69 years, sex ratio of 2.7 favoring males, and mean history of symptoms around two years before diagnosis) do not differ from previous studies [5]. Similarly, the chronic progression of symptoms we observed in our patients is a classic feature of this condition $[4,18]$.

In the present study, $53 \%$ of the patients had a clinical sensorimotor neuropathy and the presence of motor signs was not associated with the duration of the disease. We think this feature has not been emphasized previously. Indeed, in the follow-up series of Nobile-Orazio et al., disability was mainly caused by prominent ataxia, although motor signs were not mentioned [18]. Similarly, $28 \%$ of our patients had prominently sensory symptoms (without ataxia) that were sometimes painful suggesting small fiber involvement. We did not check for small fiber loss by skin biopsy, as this technique was not yet available in our center at the time of the study. Only $32 \%$ of our patients had prominent sensory ataxia. This is in contrast with previous studies where ataxia was found in more than two-thirds of patients $[6,19]$.

The pattern of nerve conduction abnormalities in antiMAG neuropathy has been widely described in the literature and most authors have emphasized the distal accentuation of nerve conduction slowing [20,21]. Moreover, consensus diagnostic criteria for anti-MAG neuropathy have been published and mostly rely on this feature along with severe reduction of SNAPs [11]. In our series, only two-thirds of patients had these typical electrodiagnostic features, while $30 \%$ of our patients had nerve conduction studies consistent with CIDP 
[12]. Moreover, most (83\%) of the patients with the CIDPlike pattern who underwent nerve biopsy did not display the classic WML on electron microscopic examination.

Widening of myelin lamellae (WML) was present in 78\% of the patients who had nerve biopsy and nerve conduction studies typical of the DADS phenotype. WML is usually observed in the outer myelin lamellae and is caused by dissociation of the intraperiod lines by the monoclonal component and is a specific feature of anti-MAG neuropathy. In our patients, WML constantly coexisted with macrophageassociated demyelination, a feature that is considered typical of CIDP although it has been described by others in patients with demyelinating neuropathy and monoclonal gammopathy [22]. One patient in this series had prominent B cell infiltrates in the epineurium, a feature that prompted a change in her treatment.

In this study, patients have been tested for the presence of anti-MAG antibodies by ELISA. Western blot (WB) analysis has long been the gold standard for detection of anti-MAG antibodies and positive $\mathrm{WB}$ is present in approximately $50 \%$ of patients with IgM monoclonal gammopathy and a demyelinating polyneuropathy [23]. The ELISA method, which is much simpler, can be applied in most immunology laboratories. Moreover, a recent work has shown that it is valid and robust in detecting anti-MAG antibodies in patients with IgM MG and polyneuropathy [13]. In this series, the ELISA test with a cut-off value of $1500 \mathrm{BTU}$ was proven more sensitive than WB (71.2\% versus $54 \%)$ to detect anti-MAG antibodies in patients with IgM MG and a demyelinating polyneuropathy. In the present study, we used a cut-off value of 1000 BTU but only 2 patients had a titer below 1500 BTU (see Table 1) so we think this has not significantly biased our results.

Results of indirect immunofluorescence were highly correlated with electrodiagnostic studies and neuropathological data in our patients. Indeed, among the ten patients who had an indirect immunofluorescence study in the CIDP group, the test was negative in seven. This was also correlated with a low titer of anti-MAG antibodies, which was below 10000 BTU in 10 out of 17 patients in this subgroup. This feature was not surprising, as other authors have previously shown that sera from patients with low titers of anti-MAG antibodies did not bind normal nerve, suggesting microheterogeneity of anti-MAG antibodies [24]. Conversely, the majority of patients with neurophysiological studies consistent with anti-MAG neuropathy and high titers of anti-MAG antibodies had a positive indirect immunofluorescence study. This simple test has been reported as highly specific even in patients with no detectable IgM gammopathy [25].

Four patients (11, 18, 23, and 29) in our series had a unique immunopathologic profile. They had a low titer of anti-MAG antibodies by ELISA (1111 to 8363 BTU) and 2 of these had a negative Western blot. These 4 patients, who had electrodiagnostic testing suggestive of anti-MAG neuropathy and typical WML for 3 of them, had positive SGPG antibody testing. This may suggest that anti-SGPG antibody testing is more sensitive to detect a relationship between the polyneuropathy and IgM monoclonal gammopathy in some patients, as previously outlined [26]. Moreover, in the recent series by
Kuijf et al., the positivity of anti-MAG antibody and antiSGPG antibody testing was highly correlated but 4 patients with a demyelinating neuropathy and IgM gammopathy had negative anti-MAG antibodies and positive anti-SGPG antibodies [13].

In our patients with high (>10000 BTU) titer of anti-MAG antibodies, nerve conduction studies were almost constantly suggestive of the DADS phenotype although the clinical picture was heterogeneous. Moreover, most of these patients had positive indirect immunofluorescence and WML. These data point to a direct relationship between the monoclonal gammopathy and the demyelinating neuropathy, by contrast with some of the patients with the CIDP-like profile. Additionally, 5 patients had positive anti-MAG antibodies by immunochromatography without quantification and could not have ELISA testing. Nevertheless, 4 of these patients had positive indirect immunofluorescence and 4 had nerve biopsy showing positive direct immunofluorescence and the presence of WML.

In summary, the data presented here clearly show the heterogeneity of polyneuropathy associated with anti-MAG antibodies. The clinical and pathological heterogeneity has already been emphasized recently [27], but we expand previous findings by demonstrating relationships between immunological, neurophysiological, and pathological profiles. In our series, clinical analysis suggests three main patterns of peripheral nervous system involvement: a sensory ataxic neuropathy, an almost purely subjective sensory (sometimes painful) neuropathy, and a sensory and motor neuropathy with variable degrees of weakness. Similarly, electrodiagnostic studies revealed that most of the patients had the phenotype referred to as DADS, although at least onethird of the patients had a CIDP-like presentation that may indicate a different pathophysiologic mechanism. Altogether, our data suggest that patients with anti-MAG neuropathy based on ELISA testing may be divided in two subgroups, according to nerve conduction studies, nerve biopsy findings, immunofluorescence data, and titers of anti-MAG antibodies. The first subgroup consists of patients with the typical anti-MAG neuropathy who have a sensory or sensorimotor polyneuropathy with demyelinating features predominating in the distal segments of nerves, prominent alterations of sensory potentials, positive immunofluorescence studies, WML, and high titers (above 8-10000 BTU) of anti-MAG antibodies. The second subgroup consists of patients with a CIDP-like neuropathy, who have a sensory or sensorimotor neuropathy with demyelinating features involving intermediate or proximal segments of nerves, sometimes preserved sensory potentials, negative immunofluorescence studies, no WML, and low titers of anti-MAG antibodies.

As the present study was retrospective, the phenotypes defined here were not taken into account at the time the patients were treated, and the care of the patients studied was considerably heterogeneous, from supportive treatment alone to the use of intravenous immunoglobulin, immunosuppressants, or monoclonal antibody (rituximab). We expect that our findings may have implications regarding the choice of therapy. Indeed, as reviewed [28], immunomodulatory treatments like intravenous immunoglobulin (IVIg) 
have demonstrated a short term and modest effect on patients with polyneuropathy and anti-MAG antibodies and are not usually considered as a first-line treatment of this condition [29]. Moreover, case reports have emphasized that a motor phenotype of anti-MAG associated neuropathy could be linked to a good response to IVIg [30]. Similarly, open-label trials have shown that plasma exchange in combination with cyclophosphamide may be beneficial in the short term [31] but does not provide any advantage over chlorambucil alone [32]. More recent studies suggest that rituximab is effective but in no more than half of the patients with polyneuropathy associated with anti-MAG antibodies [33, 34]. We think these discrepancies may be partly due to the heterogeneity of this syndrome, which may involve different pathogenic mechanisms. Deciphering these mechanisms is challenging but may help finding the best targets to treat patients with a potentially disabling disease.

\section{Conclusions}

We have described a series of patients with polyneuropathy associated with $\operatorname{IgM}$ monoclonal gammopathy and antiMAG antibodies. Anti-MAG neuropathy is regarded as a homogeneous disorder characterized by a sensory ataxic neuropathy sometimes associated with tremor [5], but our personal experience is that this syndrome is characterized by more heterogeneity than was previously reported.

This study clearly demonstrates that anti-MAG neuropathy is indeed a relatively heterogeneous entity and that this heterogeneity can be found at the clinical, neurophysiological, and pathological levels. We suggest that this heterogeneity may be underlined by different pathophysiological mechanisms that remain to be elucidated. If this assumption is true, it could have major therapeutic implications in this frequent and sometimes debilitating disorder.

\section{Ethics Approval}

Informed consent was obtained from all subjects, but this retrospective study does not require an ethics committee approval according to the current laws in our hospital.

\section{Conflict of Interests}

The authors declare that there is no conflict of interests regarding the publication of this paper.

\section{Acknowledgments}

The authors thank Marie-Odile Jauberteau-Marchand and Christine Caudie for the anti-MAG antibody testing and Martine Piaser and Laurence Richard for technical assistance.

\section{References}

[1] J. J. Kelly Jr., R. A. Kyle, P. C. O’Brien, and P. J. Dyck, "Prevalence of monoclonal protein in peripheral neuropathy," Neurology, vol. 31, no. 11, pp. 1480-1483, 1981.
[2] P. E. Braun, D. E. Frail, and N. Latov, "Myelin-associated glycoprotein is the antigen for a monoclonal IgM in polyneuropathy," Journal of Neurochemistry, vol. 39, no. 5, pp. 1261-1265, 1982.

[3] I. S. Smith, S. N. Kahn, B. W. Lacey et al., "Chronic demyelinating neuropathy associated with benign IgM paraproteinaemia," Brain, vol. 106, no. 1, pp. 169-195, 1983.

[4] N. C. Notermans, J. H. J. Wokke, H. M. Lokhorst, H. Franssen, Y. van der Graaf, and F. G. I. Jennekens, "Polyneuropathy associated with monoclonal gammopathy of undetermined significance. A prospective study of the prognostic value of clinical and laboratory abnormalities," Brain, vol. 117, no. 6, pp. 1385-1393, 1994.

[5] I. S. Smith, "The natural history of chronic demyelinating neuropathy associated with benign IgM paraproteinaemia: a clinical and neurophysiological study," Brain, vol. 117, no. 5, pp. 949-957, 1994.

[6] B. Chassande, J.-M. Léger, A. B. Younes-Chennoufi et al., "Peripheral neuropathy associated with IgM monoclonal gammopathy: correlations between M-protein antibody activity and clinical/electrophysiological features in 40 cases," Muscle and Nerve, vol. 21, no. 1, pp. 55-62, 1998.

[7] A. Vital, C. Vital, J. Julien, A. Baquey, and A. J. Steck, "Polyneuropathy associated with IgM monoclonal gammopathy. Immunological and pathological study in 31 patients," Acta Neuropathologica, vol. 79, no. 2, pp. 160-167, 1989.

[8] T. Maisonobe, B. Chassande, M. Vérin, M. Jouni, J.-M. Léger, and P. Bouche, "Chronic dysimmune demyelinating polyneuropathy: a clinical and electrophysiological study of 93 patients," Journal of Neurology Neurosurgery and Psychiatry, vol. 61, no. 1, pp. 36-42, 1996.

[9] J.-M. Vallat, F. Tabaraud, L. Magy et al., "Diagnostic value of nerve biopsy for atypical chronic inflammatory demyelinating polyneuropathy: evaluation of eight cases," Muscle \& Nerve, vol. 27, no. 4, pp. 478-485, 2003.

[10] D. Cocito, G. Isoardo, P. Ciaramitaro et al., "Terminal latency index in polyneuropathy with IgM paraproteinemia and antiMAG antibody," Muscle \& Nerve, vol. 24, no. 10, pp. 1278-1282, 2001.

[11] Joint Task Force of the EFNS and the PNS, "European Federation of Neurological Societies/Peripheral Nerve Society Guideline on management of paraproteinemic demyelinating neuropathies. Report of a Joint Task Force of the European Federation of Neurological Societies and the Peripheral Nerve Society-first revision," Journal of the Peripheral Nervous System, vol. 15, no. 3, pp. 185-195, 2010.

[12] Joint Task Force of the EFNS and the PNS, "European Federation of Neurological Societies/Peripheral Nerve Society Guideline on management of chronic inflammatory demyelinating polyradiculoneuropathy: report of a joint task force of the European Federation of Neurological Societies and the Peripheral Nerve Society-first revision," Journal of the Peripheral Nervous System, vol. 15, no. 1, pp. 1-9, 2010.

[13] M. L. Kuijf, M. Eurelings, A. P. Tio-Gillen et al., "Detection of anti-MAG antibodies in polyneuropathy associated with IgM monoclonal gammopathy," Neurology, vol. 73, no. 9, pp. 688695, 2009.

[14] J. M. Vallat, M. O. Jauberteau, D. Bordessoule, C. Yardin, P. M. Preux, and P. Couratier, "Link between peripheral neuropathy and monoclonal dysglobulinemia: a study of 66 cases," Journal of the Neurological Sciences, vol. 137, no. 2, pp. 124-130, 1996.

[15] M. O. Jauberteau, B. Younes-Chennoufi, M. Rigaud, and N. Baumann, "IGM gammopathy and polyneuropathy react with 
an antigenic glycolipid present in human central nervous system," Neuroscience Letters, vol. 97, no. 1-2, pp. 181-184, 1989.

[16] J. S. Katz, D. S. Saperstein, G. Gronseth, A. A. Amato, and R. J. Barohn, "Distal acquired demyelinating symmetric neuropathy," Neurology, vol. 54, no. 3, pp. 615-620, 2000.

[17] D. Bouhassira, N. Attal, H. Alchaar et al., "Comparison of pain syndromes associated with nervous or somatic lesions and development of a new neuropathic pain diagnostic questionnaire (DN4)," Pain, vol. 114, no. 1-2, pp. 29-36, 2005.

[18] E. Nobile-Orazio, N. Meucci, L. Baldini, A. Di Troia, and G. Scarlato, "Long-term prognosis of neuropathy associated with anti-MAG IgM M-proteins and its relationship to immune therapies," Brain, vol. 123, no. 4, pp. 710-717, 2000.

[19] E. Ellie, A. Vital, A. Steck, J.-M. Boiron, C. Vital, and J. Julien, "Neuropathy associated with 'benign' anti-myelin-associated glycoprotein IgM gammopathy: clinical, immunological, neurophysiological pathological findings and response to treatment in 33 cases," Journal of Neurology, vol. 243, no. 1, pp. 34-43, 1996.

[20] S. Attarian, J. P. Azulay, J. Boucraut, N. Escande, and J. Pouget, "Terminal latency index and modified F ratio in distinction of chronic demyelinating neuropathies," Clinical Neurophysiology, vol. 112, no. 3, pp. 457-463, 2001.

[21] D. A. Kaku, J. D. England, and A. J. Sumner, "Distal accentuation of conduction slowing in polyneuropathy associated with antibodies to myelin-associated glycoprotein and sulphated glucuronyl paragloboside," Brain, vol. 117, no. 5, pp. 941-947, 1994.

[22] A. Vital, A. Lagueny, J. Julien et al., "Chronic inflammatory demyelinating polyneuropathy associated with dysglobulinemia: a peripheral nerve biopsy study in 18 cases," Acta Neuropathologica, vol. 100, no. 1, pp. 63-68, 2000.

[23] R. H. Quarles, "Myelin-associated glycoprotein in demyelinating disorders," Critical Reviews in Neurobiology, vol. 5, no. 1, pp. $1-28,1989$.

[24] F. Fluri, F. Ferracin, B. Erne, and A. J. Steck, "Microheterogeneity of anti-myelin-associated glycoprotein antibodies," Journal of the Neurological Sciences, vol. 207, no. 1-2, pp. 43-49, 2003.

[25] J. M. Gabriel, B. Erne, L. Bernasconi et al., "Confocal microscopic localization of anti-myelin-associated glycoprotein autoantibodies in a patient with peripheral neuropathy initially lacking a detectable IgM gammopathy," Acta Neuropathologica, vol. 95, no. 5, pp. 540-546, 1998.

[26] C. Caudie, F. Bouhour, P. Petiot et al., "Contribution des autoanticorps IgM anti-SGPG par Elisa Bülhmann au diagnostic de 147 neuropathies périphériques démyélinisantes associées à une IgM monoclonale," Annales de Biologie Clinique (Paris), vol. 65, no. 4, pp. 369-375, 2007.

[27] M. Luigetti, A. Conte, N. Montano et al., "Clinical and pathological heterogeneity in a series of 31 patients with IgM-related neuropathy," Journal of the Neurological Sciences, vol. 319, no. 12, pp. 75-80, 2012.

[28] L. Magy and J.-M. Vallat, "Evidence-based treatment of chronic immune-mediated neuropathies," Expert Opinion on Pharmacotherapy, vol. 10, no. 11, pp. 1741-1754, 2009.

[29] G. Comi, L. Roveri, A. Swan et al., "A randomised controlled trial of intravenous immunoglobulin in IgM paraprotein associated demyelinating neuropathy," Journal of Neurology, vol. 249, no. 10, pp. 1370-1377, 2002.

[30] Y. Kawagashira, N. Kondo, N. Atsuta et al., "IgM MGUS antiMAG neuropathy with predominant muscle weakness and extensive muscle atrophy," Muscle and Nerve, vol. 42, no. 3, pp. 433-435, 2010.
[31] G. Blume, A. Pestronk, and L. T. Goodnough, "Anti-MAG antibody-associated polyneuropathies: improvement following immunotherapy with monthly plasma exchange and IV cyclophosphamide," Neurology, vol. 45, no. 8, pp. 1577-1580, 1995.

[32] E. Oksenhendler, S. Chevret, J. Leger et al., "lasma exchange and chlorambucil in polyneuropathy associated with monoclonal IgM gammopathy. IgM-associated Polyneuropathy Study Group," Journal of Neurology Neurosurgery and Psychiatry, vol. 59, no. 3, pp. 243-247, 1995.

[33] M. C. Dalakas, G. Rakocevic, M. Salajegheh et al., "Placebocontrolled trial of rituximab in IgM anti-myelin-associated glycoprotein antibody demyelinating neuropathy," Annals of Neurology, vol. 65, no. 3, pp. 286-293, 2009.

[34] J.-M. Léger, K. Viala, G. Nicolas et al., "Placebo-controlled trial of rituximab in IgM anti-myelin-associated glycoprotein neuropathy," Neurology, vol. 80, no. 24, pp. 2217-2225, 2013. 


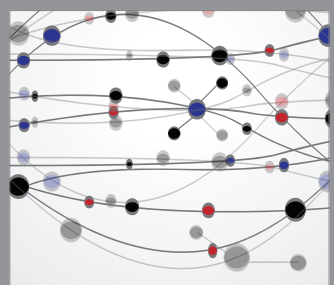

The Scientific World Journal
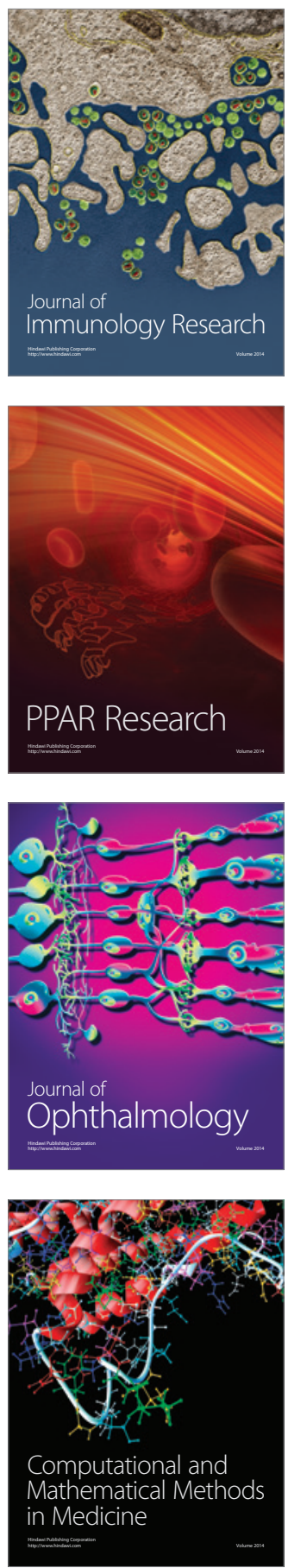

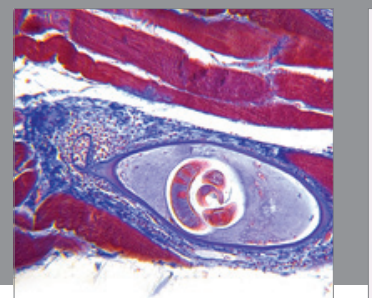

Gastroenterology

Research and Practice
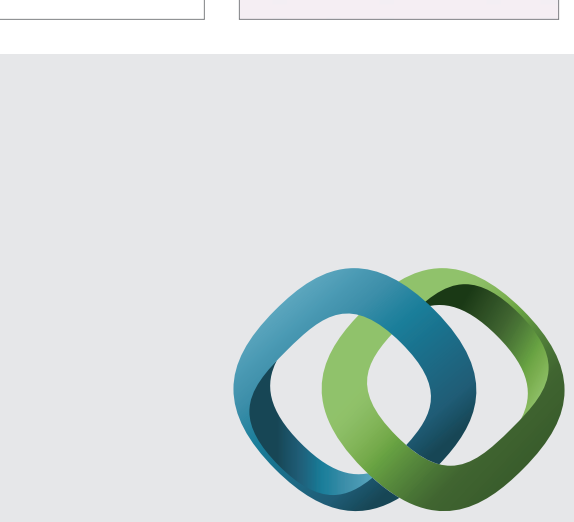

\section{Hindawi}

Submit your manuscripts at

http://www.hindawi.com
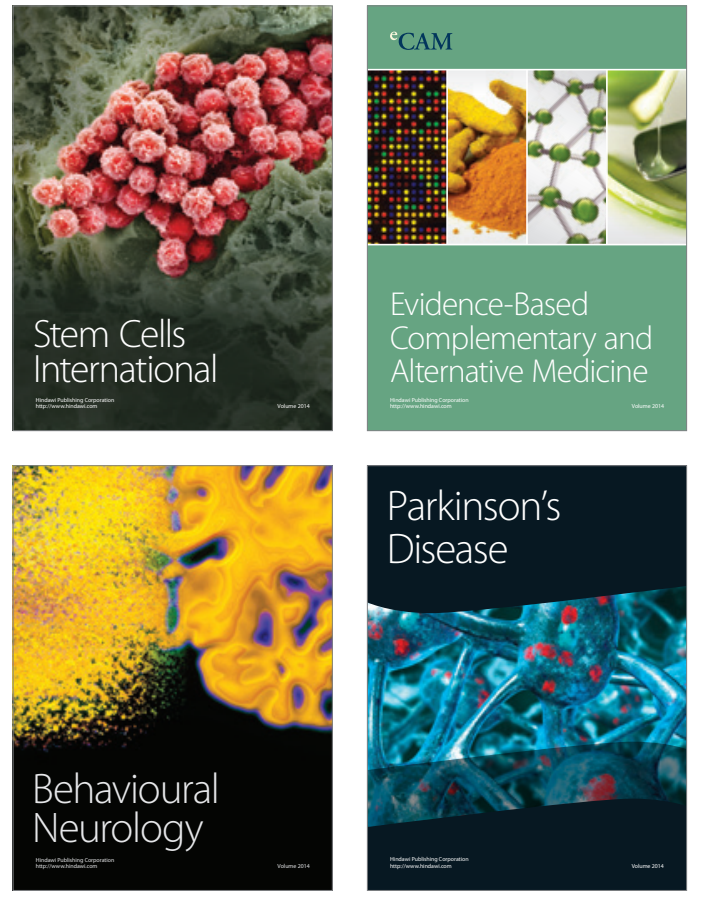
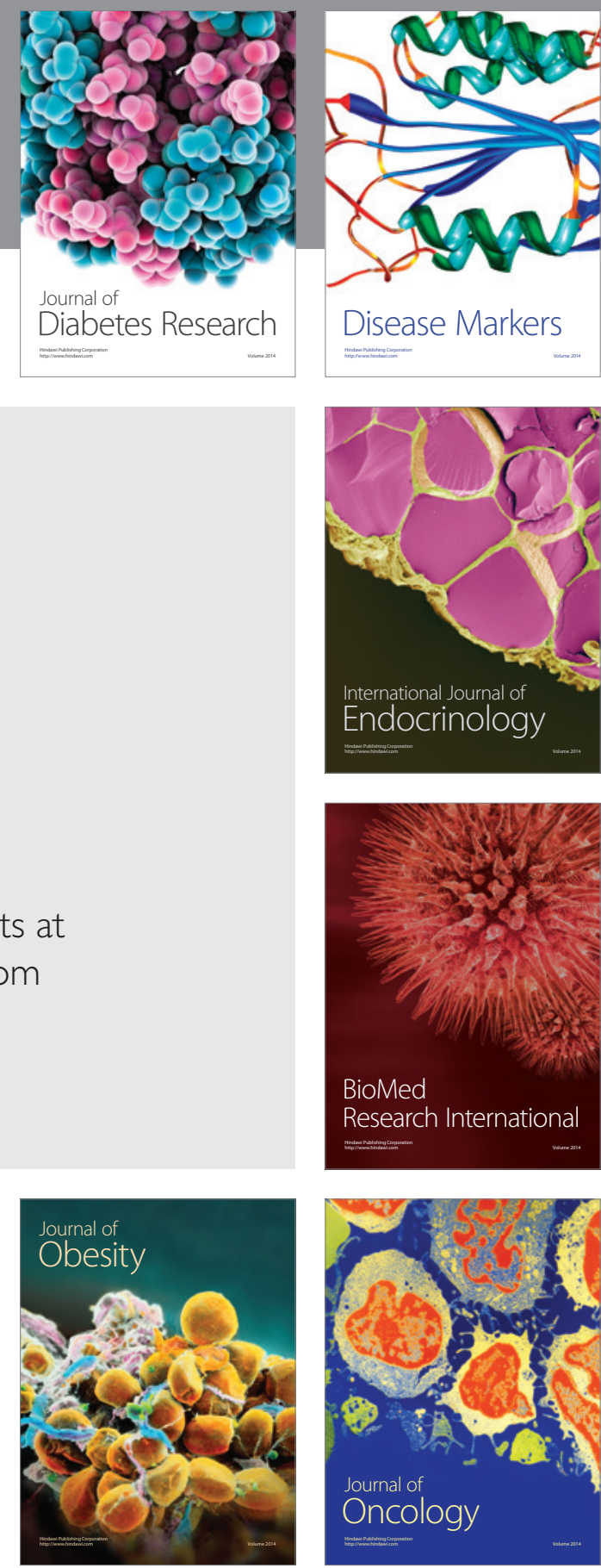

Disease Markers
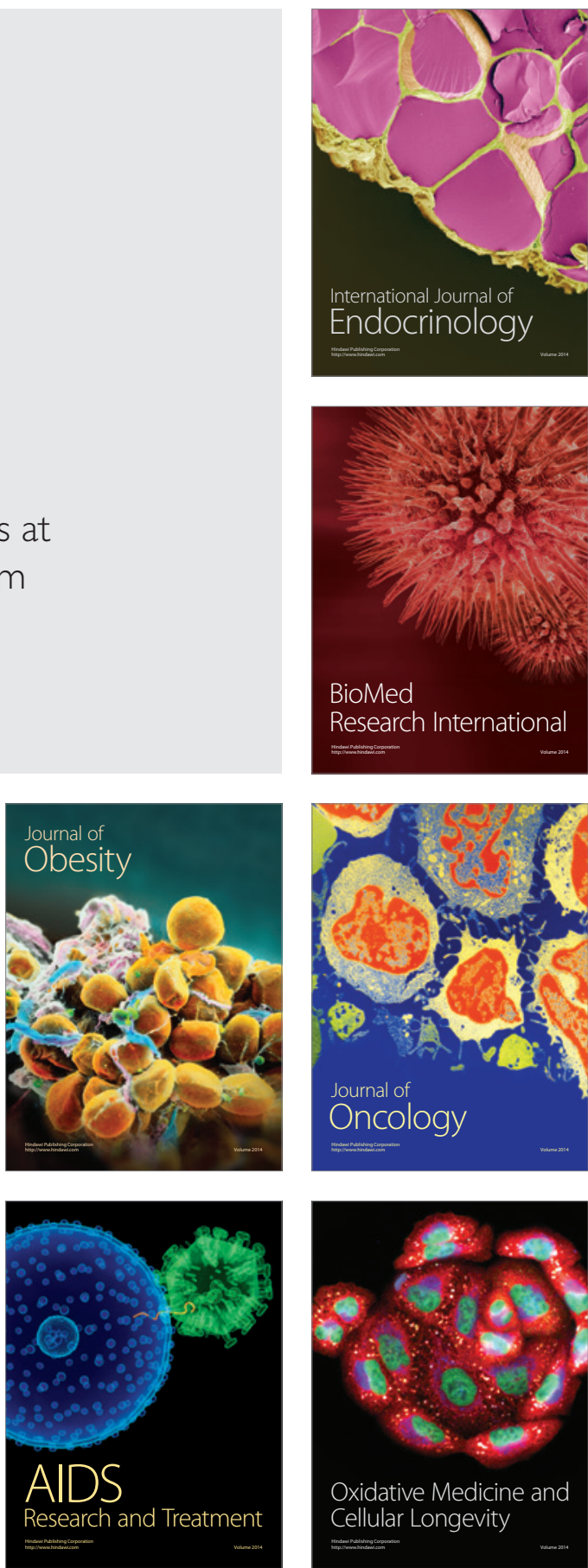